\title{
ANÁlISE QUANTITATIVA SOBRE GRAVIDEZ NA ADOLESCÊNCIA EM UM MUNICÍPIO MINEIRO*
}

Suelen Souza do Carmo', Darlan Einstein do Livramento², Hipólito Ferreira Paulino Neto³, Mariana Gondim Mariutti Zeferino ${ }^{4}$

${ }^{1}$ Enfermeira. Hospital Municipal Dr. Mário Gatti. Campinas-SP-Brasil.

${ }^{2}$ Engenheiro Agrônomo. Doutor em Agronomia. Libertas Faculdades Integradas. São Sebastião do Paraíso-MG-Brasil.

${ }^{3}$ Biólogo. Pós-doutor em Entomologia. Universidade de São Paulo. São Sebastião do Paraíso-MG-Brasil.

${ }^{4}$ Enfermeira. Doutora em enfermagem. Universidade de São Paulo e Libertas Faculdades Integradas. Ribeirão Preto-SP-Brasil.

RESUMO: Estudo quantitativo e descritivo, que objetivou verificar frequência, causas e consequências da gravidez na adolescência. Foram entrevistadas 104 adolescentes cadastradas no Sistema de Informações sobre Nascidos Vivos em 2010, que se encontravam na faixa etária dos 15 aos 21 anos; a maioria apresentava baixa escolaridade; $60 \%$ não trabalhavam; todas realizaram pré-natal; foram observadas alterações no estado conjugal após a gestação e aumento no conhecimento e uso dos métodos contraceptivos; $74 \%$ não planejaram a gravidez; relataram mudanças após a gravidez como maior responsabilidade (26\%) e perda da liberdade (23\%). Concluiuse ser necessária a aproximação com as adolescentes para esclarecer dúvidas, desprender-se de preconceitos, realizar assistência holística com orientações objetivas, corretas e claras, respeitando crenças e individualidades. DESCRITORES: Gravidez na adolescência; Enfermagem; Saúde da mulher; Saúde pública.

\section{QUANTITATIVE ANALYSIS REGARDING PREG- NANCY IN ADOLESCENCE IN A MUNICIPALITY IN MINAS GERAIS}

\begin{abstract}
This quantitative and descriptive study aimed to assess the frequency, causes, and consequences of pregnancy in adolescence. Interviews were held with 104 adolescents registered on the Live Births Information System in 2010, who were in the age range of $15-21$ years old; the majority had low educational levels; $60 \%$ were not working; all attended prenatal care; changes were observed in their conjugal status after the pregnancy, as was increase in their knowledge and use of methods of contraception; $74 \%$ did not plan the pregnancy; and they reported changes following the pregnancy such as greater responsibility $(26 \%)$ and loss of freedom $(23 \%)$. It is concluded that it is necessary to become closer to the adolescents so as to clarify doubts, to break away from one's own prejudices, and to provide holistic care with objective, correct and clear guidance, respecting beliefs and individuality.
\end{abstract}

DESCRIPTORS: Pregnancy in adolescence; Nursing; Women's health; Public health.

\section{ANÁLISIS CUANTITATIVO SOBRE GRAVIDEZ EN LA ADOLESCENCIA EN UN MUNICIPIO DE MINAS GERAIS}

RESUMEN: Estudio cuantitativo y descriptivo cuya finalidad fue verificar frecuencia, causas y consecuencias de la gravidez en la adolescencia. Fueron entrevistadas 104 adolescentes registradas en el Sistema de Informaciones sobre Nacidos Vivos en 2010, que se encontraban en la franja etaria de los 15 a los 21 años; la mayoría presentava baja escolaridad; $60 \%$ no trabajaban; todas realizaron prenatal; fueron observadas alteraciones en el estado conyugal después de la gestación y aumento en el conocimiento y uso de los métodos anticonceptivos; $74 \%$ no planeaban la gravidez; relataron cambios después de la gravidez como mayor responsabilidad (26\%) y pérdida de libertad (23\%). Se concluye que es necesaria una aproximación con las adolescentes para aclarar dudas, libertarse de prejuicios, realizar asistencia holística con orientaciones objetivas, correctas y claras, respetando creencias y individualidades.

DESCRIPTORES: Gravidez en la adolescencia; Enfermería; Salud de la mujer; Salud pública.

\footnotetext{
*Artigo extraído da Monografia de conclusão de Curso de Enfermagem intitulada: Frequência, causas e consequências para a gravidez na adolescência em São Sebastião do Paraíso-MG. Libertas Faculdades Integradas. São Sebastião do Paraíso-MG, 2012.
} 


\section{INTRODUÇÃO}

A adolescência é um período de transição entre a infância e a fase adulta, com mudanças físicas e psicológicas, que acarreta a estes indivíduos diversos tipos de comportamentos que podem variar de acordo com a família, a religião, a cultura, a nacionalidade e a ideologia de cada indivíduo ${ }^{(1)}$.

Segundo a Organização Mundial da Saúde e a UNICEF (Fundo das Nações Unidas para a Infância) ${ }^{(2)}$, cronologicamente é adolescente o jovem de faixa etária entre 10 e 19 anos, podendo ser subdividido em duas etapas - fase inicial da adolescência (dos 10 aos 14 anos de idade) e fase final da adolescência (dos 15 aos 19 anos de idade). Nesta fase a sexualidade do adolescente é extremamente impetuosa, o jovem não se preocupa com as consequências de seus atos, pensa somente no prazer do momento, o que muitas vezes leva à gravidez precoce ${ }^{(1,3)}$.

No Brasil, 17 milhões de pessoas pertencem ao sexo feminino com idade entre 10 e 19 anos $^{(4)}$ e nascem mais de um milhão de crianças de mães adolescentes por ano ${ }^{(5)}$. Acredita-se que vários aspectos estão envolvidos nesse grande número de casos de gravidez na adolescência: influência dos meios de comunicação e da mídia, diminuição de tabus e inibições sexuais, falta de diálogo com pais e professores, desestruturação familiar, adiantamento da menarca, autoafirmação social e, finalmente, a gravidez sendo considerada como rito de passagem da adolescência para a fase adulta ${ }^{(3,5)}$.

Contudo, apesar da existência de orientações nos diversos tipos de mídia e de distribuição gratuita de métodos contraceptivos, o índice de gravidez na adolescência no Brasil continua alto, sendo necessária uma maior conscientização da população alvo. Além disso, uma compreensão melhor da realidade e das necessidades dos adolescentes possibilitaria elaborar campanhas mais objetivas, eficazes e efetivas ${ }^{(3,5)}$.

Assim, este trabalho teve como objetivos principais analisar quantitativamente a gravidez na adolescência em um município mineiro, sua frequência, bem como as principais causas para o acontecimento da gravidez na adolescência e consequências para a vida destas adolescentes.

\section{METODOLOGIA}

Trata-se de um estudo quantitativo descritivo, desenvolvido no município de São Sebastião do Paraíso - MG no ano de 2012. A população de estudo constituiu-se de todas as mães adolescentes com idade entre 10 a 19 anos (completos na data do parto) segundo classificação etária de adolescência da OMS, que tiveram a Declaração de Nascido Vivo (DNV) preenchida e disponível no Sistema de Informação de Nascidos Vivos (SINASC).

Os dados do SINASC do período de janeiro a outubro do ano de 2010 foram obtidos através da Vigilância Epidemiológica da Secretaria de Saúde e Ação Social do Município de São Sebastião do Paraíso - $M G^{(6)}$. Foram selecionados somente sujeitos com idade entre 10 a 19 anos, totalizando 150 cadastrados. Entretanto quatro cadastros se repetiam devido ao nascimento de gêmeos, havendo duas DNV para cada uma destas mães e nove adolescentes não eram cadastradas no sistema de cadastros de serviços de saúde da Secretaria de Saúde e Ação Social do município, resultando uma nova amostra de 137 adolescentes, as quais foram contatadas diretamente em seu domicílio para conhecerem a proposta da pesquisa e serem convidadas à participação do estudo. Entretanto, 12 adolescentes haviam se mudado do município; uma era presidiária; duas se recusaram a participar do estudo e 18 delas foram impossíveis de ser localizadas, resultando a amostra final em 104 adolescentes entrevistadas.

O instrumento de coleta de dados foi analisado, revisto e aprovado por quatro profissionais, sendo eles uma Doutora em Enfermagem, uma Mestra em Enfermagem, uma Doutora em Psicologia e um Assistente Social. As variáveis contidas no formulário compreendiam as possíveis causas e consequências da gravidez precoce destas adolescentes.

A coleta de dados foi realizada de dezembro de 2011 a março de 2012. A abordagem foi direta com cada jovem. Os dados foram coletados, por meio de entrevista com duração de aproximadamente 30 minutos.

Este estudo esteve de acordo com as orientações éticas da Resolução 196/96 do Conselho Nacional de Saúde (CNS), que regulamenta pesquisas envolvendo seres 
humanos. Foi submetido à aprovação do Comitê de Ética em Pesquisa da Escola de Enfermagem de Ribeirão Preto - Universidade de São Paulo sob Protocolo $n^{\circ}$ 1420/2011. Adicionalmente, obtevese autorização da Coordenadora da Atenção Primária de Saúde do município de São Sebastião do Paraíso - Minas Gerais para realização da pesquisa. O Termo de Consentimento Livre e Esclarecido (TCLE) foi assinado por cada um dos sujeitos da pesquisa (quando menores de 18 anos foi registrado também a anuência de seu responsável legal), e cada participante recebeu uma cópia deste termo.

\section{RESULTADOS}

A faixa etária das 104 adolescentes estudadas variou de 15 a 21 anos, a maioria (61\%) apresentando entre dezoito e vinte anos, com idade média de 18,5 $\pm 1,64$ anos $(S ; n=104$ adolescentes). As adolescentes, em sua maioria, possuíam Ensino Fundamental incompleto (37\%), 16\% tinham Ensino Fundamental completo, 27\% tinham Ensino Médio incompleto, 18\% tinham Ensino Médio completo e 2\% delas tinham Ensino Superior incompleto, sendo que do total $(n=104)$, apenas 13\% das entrevistadas estudavam à época da realização deste estudo.

Praticamente metade da amostra (49\%) encontrava-se em relações de união estável, $20 \%$ namoravam, 15\% estavam casadas, $15 \%$ estavam solteiras e $1 \%$ delas estava desquitada. Em relação à profissão das jovens mães, 60\% não trabalhavam naquele momento, $21 \%$ pertenciam ao setor de produção, 9\% delas eram atendentes e 10\% das entrevistadas ocupavam outros cargos.

Em relação aos dados obstétricos, observouse que a maioria das adolescentes tinha somente um filho (84\%), o fruto dessa gestação em estudo, $12 \%$ tinham dois filhos e $4 \%$ delas haviam gerado três filhos. A quantidade média de filhos foi de 1,2 $\pm 0,49$ (S; $n=104$ adolescentes). Das adolescentes entrevistadas, 9\% estavam gestantes novamente. Um total de 95\% delas nunca havia sofrido aborto, enquanto 5\% delas já haviam passado por esse episódio.

Em relação à idade à época de início da vida sexual, 32\% das entrevistadas tinham 15 anos, 17\% com 13 anos, 16\% com 14 anos, outros 16\% delas com 16 anos, 9\% com 17 anos, 6\% com 12 anos e 4\% com 18 anos de idade. A idade média foi de 14,8 $\pm 1,48$ anos ( $S ; n=104$ adolescentes).

Da amostra pesquisada 38\% das adolescentes não tinham diálogo sobre métodos contraceptivos com ninguém, $27 \%$ com familiares, $22 \%$ com amigas, $10 \%$ com o parceiro e $3 \%$ delas conversavam sobre o assunto com outros.

Todas as adolescentes fizeram pré-natal durante suas gestações, $86 \%$ destes procedimentos foram realizados pelo Sistema Único de Saúde (SUS), 10\% deles foram realizados por convênios de saúde particulares e os 4\% restantes foram realizados juntamente pelos dois tipos de serviços citados anteriormente. Em relação ao parto, 98\% deles foram realizados pelo SUS e somente $2 \%$ deles foram pelo atendimento particular.

Foi registrado um aumento no conhecimento das jovens mães sobre métodos contraceptivos após a situação de gravidez conforme mostra a Tabela 1.

Tabela 1 - Conhecimento e uso de métodos contraceptivos pelas adolescentes antes de engravidar e atualmente. São Sebastião do Paraíso-MG-Brasil, dezembro 2011 a março 2012.

\begin{tabular}{lcccc}
\hline $\begin{array}{l}\text { Método } \\
\text { Contraceptivo }\end{array}$ & $\begin{array}{c}\text { Conhecimento antes } \\
\text { de engravidar }\end{array}$ & $\begin{array}{c}\text { Conhecimento no mo- } \\
\text { mento da entrevista }\end{array}$ & $\begin{array}{l}\text { Uso antes de } \\
\text { engravidar }\end{array}$ & \multicolumn{2}{c}{$\begin{array}{c}\text { Uso no momento da } \\
\text { entrevista }\end{array}$} \\
\hline Nenhum & $1 \%$ & $2 \%$ & $57 \%$ & $31 \%$ \\
\hline Camisinha & $98 \%$ & $98 \%$ & $18 \%$ & $9 \%$ \\
\hline Tabelinha & $43 \%$ & $50 \%$ & $2 \%$ & $1 \%$ \\
\hline Pílula anticoncepcional & $97 \%$ & $98 \%$ & $26 \%$ & $49 \%$ \\
\hline Coito interrompido & $36 \%$ & $42 \%$ & $3 \%$ & $0 \%$ \\
\hline $\begin{array}{l}\text { Injeção } \\
\text { anticoncepcional }\end{array}$ & $75 \%$ & $86 \%$ & $2 \%$ & $13 \%$ \\
\hline DIU & $57 \%$ & $62 \%$ & $0 \%$ & $0 \%$ \\
\hline Pílula de emergência & $66 \%$ & $70 \%$ & $3 \%$ & $0 \%$ \\
\hline Outro & $5 \%$ & $5 \%$ & $0 \%$ & $0 \%$ \\
\hline
\end{tabular}


Das adolescentes que alegaram utilizar métodos contraceptivos antes de engravidar, 38\% das mesmas referiram usar sempre, enquanto $62 \%$ usavam às vezes, ou com falhas. Um total de 71 adolescentes fazia uso de um ou mais métodos contraceptivos no momento da pesquisa e todas afirmaram que utilizavam estes métodos de modo correto e sem cessar.

Das entrevistadas, 23\% tinham 17 anos de idade na época do parto, 21\% tinham 18 anos, $17 \%$ com 19 anos, $16 \%$ com 16 anos, $13 \%$ com 15 anos, $8 \%$ com 14 anos e $2 \%$ delas tinham 13 anos de idade, com idade média de 16,8 \pm 1,60 anos (S; n=104 adolescentes).

Foi possível observar também alterações no estado civil das adolescentes entrevistadas. Quando engravidaram, a maioria delas, 63\%, namoravam, $23 \%$ delas mantinham união estável, 9\% eram casadas e $5 \%$ estavam solteiras (sozinhas). Ao término do estudo, a maioria manteve união estável, um total de $49 \%$, 20\% namoravam, 16\% estavam casadas e $15 \%$ delas estavam solteiras (sozinhas). Observou-se, ainda, que $75 \%$ das adolescentes mantinham relacionamento afetivo com o mesmo parceiro, enquanto $25 \%$ delas não mantinham mais relacionamento com o pai de seu filho.

Das adolescentes entrevistadas, $47 \%$ já haviam interrompido os estudos antes de engravidar, 17\% já haviam completado o Ensino Médio, $32 \%$ interromperam os estudos por causa da gravidez e 4\% delas continuaram a estudar após o parto.

A maioria, 69\%, não trabalhava quando engravidou. Daquelas quetrabalhavam $(n=32), 62 \%$ retornaram ao emprego após a Licença Maternidade.

Um total de $26 \%$ das adolescentes entrevistadas assumiu ter planejado a gestação. Finalizando, das adolescentes entrevistadas, $42 \%$ delas alegaram não ter ocorrido nenhuma mudança em sua vida após a gestação. Das 58\% que relataram mudanças $(n=60), 26 \%$ relataram responsabilidade aumentada, $23 \%$ relataram perda da liberdade, $12 \%$ alegaram amadurecimento, $6 \%$ disseram ter gastos financeiros maiores, 5\% afirmaram ter a felicidade aumentada, 4\% delas disseram ter horários mais rígidos, 2\% afirmaram ter preocupação maior e $29 \%$ delas relataram outras mudanças.

\section{DISCUSSÃO}

O presente estudo mostra que a maioria das adolescentes entrevistadas possuía Ensino
Fundamental incompleto e Ensino Médio incompleto. Chama atenção o fato de que apenas 13\% delas continuaram estudando. Em estudo realizado num hospital universitário no Rio de Janeiro com 112 adolescentes gestantes, 69,6\% destas tinham Ensino Fundamental completo e 22,3\% possuíam Ensino Médio incompleto(7). Das adolescentes com reincidência de gravidez atendidas pelo setor público de Indaiatuba (SP), apenas $3,12 \%$ ainda estudavam ${ }^{(8)}$.

Estes dados indicam que baixa escolaridade está diretamente relacionada à gravidez na adolescência. A maior parte das adolescentes deste estudo havia abandonado a escola antes de engravidar. Das adolescentes que interromperam os estudos por causa da gravidez, apenas $27 \%$ delas retornaram à escola. Em pesquisa realizada pela Universidade Federal da Paraíba com 85 adolescentes puérperas, $73,8 \%$ afirmaram ser a gravidez o motivo da interrupção dos estudos ${ }^{(9)}$.

Em especial, a adolescente grávida, que muitas vezes depende economicamente da sua família, se vê na necessidade de abandonar os estudos. Ela é frequentemente abandonada pelo pai de seu bebê e às vezes por seu círculo de amizade. Ainda, apresenta baixa autoestima e se sente ressentida por ter provocado raiva e estresse em seu meio familiar. Este e outros fatores causam uma sobrecarga emocional e social, que pode resultar em problemas psicossociais que seriam evitados caso houvesse prevenção da gestação $^{(2-3)}$.

Praticamente metade das mães adolescentes se encontrava em união estável com seus parceiros no momento da entrevista. Em amostra composta por adolescentes moradoras de Indaiatuba (SP), com reincidência de gravidez, 78,12\% delas viviam em união estável ${ }^{(8)}$. Em estudo realizado pela Universidade Federal da Paraíba, com adolescentes puérperas, 83,5\% das entrevistadas referiam estar casadas ou com companheiro ${ }^{(9)}$. São comuns as alterações no estado civil das jovens grávidas, as quais muitas vezes namoravam quando engravidaram. A gravidez parece ser o que as leva a estabelecer união estável ou a se casar, visando proporcionar melhor qualidade de vida para a criança que irá nascer ${ }^{(1-3)}$.

Dentre as adolescentes do presente estudo, a maioria não trabalhava ou exercia atividades tais como auxiliar de produção e atendente. Pesquisa realizada com 85 adolescentes puérperas da 
Paraíba aponta que 92,4\% das entrevistadas não estavam trabalhando no momento da pesquisa $^{(9)}$. A pouca idade, em conjunto com a baixa escolaridade das adolescentes, reduz a probabilidade de inserção no mercado de trabalho, que exige cada vez mais capacitação e experiência. Estas exigências de mercado raramente podem ser encontradas em mães adolescentes. Adicionalmente, as que conseguem empregos são mal remuneradas, o que as deixa dependentes financeiramente de familiares e/ou parceiro $^{(1-3)}$.

A frequência de gravidez na adolescência em São Sebastião do Paraíso-MG, no período estudado, foi de 19\%, praticamente a mesma frequência nacional que, em 2011, foi de 19,25\% de nascidos vivos filhos de adolescentes de 10 a 19 anos de idade ${ }^{(10)}$. Porém este resultado foi superior à proporção de nascidos vivos de mães adolescentes em Minas Gerais que, segundo dados do Ministério da Saúde, foi de 16,48\% em $2011^{(10)}$. Em Indaiatuba, interior de São Paulo, no ano de 2008, a frequência de gravidez na adolescência foi de $30,42 \%{ }^{(8)}$. Observa-se que em algumas cidades a frequência de gravidez na adolescência está abaixo da média nacional e em outras acima, indicando que determinados locais podem ter enfrentamentos mais resolutos e eficazes enquanto outros ainda estão se desenvolvendo e aprimorando em relação a essa problemática.

Neste estudo, a idade média do início da vida sexual das adolescentes foi de 14,8 anos. Em pesquisa realizada com adolescentes de uma Escola Estadual da Zona Sul de São Paulo, o início da vida sexual dos pesquisados foi em média de 13,5 anos de idade ${ }^{(11)}$.

Em relação aos dados obstétricos, a maior parte da amostra teve somente um filho. Da totalidade de adolescentes entrevistadas durante o pré-natal realizado em um hospital universitário do Rio de Janeiro, 79,4\% delas estavam gestantes do primeiro filho(7). O presente estudo está em concordância com o estudo citado acima, apesar de apontar uma diminuição nesse valor devido aos 9\% desta amostra ter se encontrado gestante novamente no momento da entrevista. Observou-se que a adolescente que engravidou precocemente procurou se prevenir de forma eficaz, evitando assim outra gestação. Isso parece mostrar que a experiência com a gravidez ou o amadurecimento devido ao aumento da idade fez com que grande parte das adolescentes ficasse mais atenta à prevenção da gravidez.

Muitas vezes, ao se descobrir grávida, a adolescente opta pela interrupção da gravidez por meio de aborto, por não ter condições de assumir a maternidade. Outras vezes, o corpo da adolescente não está completamente preparado para a gestação e isto acaba por acarretar um aborto natural, que pode gerar consequências psicológicas traumáticas para ela ${ }^{(6)}$. Neste estudo observou-se que a minoria das adolescentes sofreu aborto, sem especificação do tipo de aborto. Em estudo feito no Rio de Janeiro, $8 \%$ das adolescentes tiveram aborto ${ }^{(7)}$. Já das adolescentes com reincidência de gestação, 25\% delas sofreram aborto ${ }^{(12)}$.

Em estudo realizado em Curitiba, Paraná, a idade média das adolescentes gestantes foi de 15 anos $^{(13)}$. Em outro estudo, a idade média das 85 adolescentes internadas na enfermaria de puerpério de um hospital universitário da Paraíba era de 18,2 anos de idade ${ }^{(9)}$. Neste presente estudo a idade média das adolescentes ao engravidar era de 16,8 anos, semelhante à média nacional, na qual a maior frequência de partos em adolescentes aconteceu na faixa etária dos 15 aos 19 anos de idade, num total de 20,6\% dos partos realizados no ano de 2006 contra 0,9\% entre adolescentes de 10 a 14 anos $^{(14)}$.

Este estudo indicou que todas as adolescentes realizaram o pré-natal, porém não foi questionada a quantidade de consultas realizadas nem a idade gestacional na época do início das consultas. Durante o pré-natal de adolescentes gestantes, atendidas em um hospital universitário do Rio de Janeiro, apurou-se que 70,5\% das adolescentes iniciaram o pré-natal no segundo trimestre de gestação, apenas 26,8\% delas deram início ao acompanhamento no primeiro trimestre ${ }^{(7)}$. Em estudo realizado em maternidade pública do município do Rio de Janeiro, com dados de 542 puérperas adolescentes, a idade gestacional média ao iniciarem o pré-natal foi de 15 semanas $^{(15)}$.

Este estudo apurou um aumento no nível do conhecimento das adolescentes sobre métodos contraceptivos após a gestação, sendo a camisinha masculina e a pílula anticoncepcional os métodos mais conhecidos. Estudo realizado em São Paulo (SP) verificou que os métodos mais conhecidos pelos jovens são a camisinha feminina (88\%), 
a camisinha masculina (70,6\%), a pílula do dia seguinte (32\%) e a pílula anticoncepcional $(26,6 \%)$ (16). Adicionalmente, gestantes adolescentes reincidentes declararam que todas conheciam algum tipo de método anticoncepcional, porém isso não foi o suficiente para a prevenção da gravide $z^{(8)}$. Pode-se dizer que isso ocorre devido ao fato de a adolescência ser uma fase na qual o indivíduo realiza ações movidas pela impulsividade, não pensando nas consequências de seus atos. Portanto, mesmo recebendo orientações sobre métodos contraceptivos, modo correto do uso e a importância do mesmo, o adolescente ainda se relaciona sem prevenção alguma de riscos.

Neste contexto, vale ressaltar que nesta pesquisa houve diferenças entre o uso de métodos contraceptivos pelas adolescentes antes e depois de engravidarem. Antes de engravidar a minoria utilizava algum método contraceptivo de modo correto e eficaz, após a gravidez houve aumento no número de adolescentes que passou a utilizar pílula anticoncepcional e a injeção anticoncepcional, de modo contínuo e adequado. Um estudo realizado com 487 adolescentes em Porto Alegre, RS, mostrou que das adolescentes que tinham vida sexual ativa, $75 \%$ referiam uso de algum método contraceptivo, em sua maioria $(61,8 \%)$ a pílula anticoncepcional ${ }^{(17)}$.

Quando questionada a respeito de diálogo sobre métodos contraceptivos, grande parte da amostra (38\%) relatou não ter diálogo com ninguém sobre o assunto. E quando há diálogo, estes se dão com familiares, amigas, parceiro e outros. Em pesquisa realizada em escola pública da zona sul de São Paulo, sobre contracepção na adolescência, 44\% dos adolescentes entrevistados relataram ter diálogo sobre métodos contraceptivos e sobre sexo com os pais, 29,3\% com amigos, $21,3 \%$ com professores e $5,4 \%$ deles afirmaram receber informação através de outras fontes ${ }^{(16)}$, indicando a existência de 'tabus' ou mesmo despreparo das pessoas em relação a dar orientações a eles sobre sexo.

Neste sentido, ao elaborar estratégias de aproximação dos adolescentes, tantos os pais e familiares, como educadores, profissionais de saúde e toda a população deve considerar as diferenças individuais e culturais de cada adolescente, para assim atenderem às expectativas e necessidades específicas tanto em relação às adolescentes que possuem parceiro sexual, quanto em relação àquelas que ainda não iniciaram sua vida sexual ${ }^{(1,3)}$.

No presente estudo, a maioria das adolescentes argumentou ter engravidado por descuido ou acidente. Uma pesquisa realizada num serviço de atenção secundária destinado a tratar da saúde materno-infantil, situado no Município de Barbalha-CE, apurou que $70 \%$ das mães adolescentes englobadas na pesquisa não planejaram a gravidez ${ }^{(18)}$. Num hospital universitário do Rio de Janeiro, que presta serviço de pré-natal a adolescentes gestantes, foi constatado que não houve planejamento da gravidez em $75 \%$ dos $\operatorname{casos}^{(7)}$. Em estudo realizado com adolescentes gestantes, moradoras de uma favela em São Paulo, 50\% não planejou a gestação ${ }^{(19)}$. Isso mostra que a maioria de adolescentes que engravidou não utilizou nenhum método contraceptivo, apesar de saberem os riscos aos quais estavam propensas.

Quando as adolescentes foram questionadas sobre mudanças percebidas em sua vida após a gravidez, 42\% delas alegaram não haver nenhuma. Para as outras, as principais mudanças foram maior responsabilidade, perda da liberdade e amadurecimento. Uma pesquisa realizada com residentes da Favela do Moinho, localizada em São Paulo, mostrou que $100 \%$ da amostra afirmou não ter havido alterações em sua vida após a gestação ${ }^{(19)}$. Já em pesquisa realizada em hospital-maternidade de Fortaleza-CE, a maioria da amostra relata inúmeras alterações em suas vidas após a gravidez ${ }^{(20)}$.

Este estudo mostrou que a maioria das adolescentes mantém relacionamento afetivo com o pai de seu filho (namoro, união estável ou casamento). Em pesquisa realizada em um Serviço de Adolescentes da Maternidade-Escola Assis Chateaubriand (MEAC), da Universidade Federal do Ceará, 80\% das adolescentes não permaneceram com o mesmo companheiro ${ }^{(21)}$.

\section{CONCLUSÃO}

As equipes de saúde e sociedade em geral devem realizar o aconselhamento anticoncepcional do adolescente com o objetivo de compreender as percepções corretas e incorretas que eles possuem acerca dos métodos contraceptivos, ajudá-los a entender o risco da atividade sexual desprotegida em seu contexto 
pessoal, explicitando os tipos de métodos contraceptivos, o funcionamento de cada um deles, o modo correto da utilização, a eficácia, as indicações e contraindicações e a atuação dupla da camisinha na prevenção da gravidez precoce e das doenças sexualmente transmissíveis, sempre dando abertura para dúvidas e se mostrando disponíveis para futuros questionamentos, tornando assim eficiente a prevenção à gravidez na adolescência e suas consequências.

\section{REFERÊNCIAS}

1. Nascimento MAL, Figueiredo NMA, Francisco MTR. Cuidados para adolescentes. In: Figueiredo NMA, organizador. Ensinando a cuidar da criança. São Caetano do Sul (SP): Yendis; 2010. p. 45-78.

2. Fundo das Nações Unidas para a infância (UNICEF). Situação Mundial da Infância 2011: Adolescência - uma fase de oportunidades. New York: UNICEF; 2011. 148 p.

3. Carvalho GM, Merighi MAB. Gravidez e maternidade precoce. In: Carvalho GM, Merighi MAB. Gravidez precoce: que problemaéesse? São Paulo(SP): Paulus; 2006. p. 33-46.

4. Instituto Brasileiro de Geografia e Estatística (IBGE). Censo 2010: distribuição da população por sexo, segundo os grupos de idade. [Internet]. 2010 [acesso em: 28 mai 2014]. Disponível: http://www.censo2010. ibge.gov.br/sinopse/webservice/default.php?cod1=0\& $\operatorname{cod} 2=\& \operatorname{cod} 3=0 \&$ frm $=$ piramide

5. Instituto Brasileiro de Geografia e Estatística (IBGE). Censo Demográfico 2010: Mulheres de 10 anos ou mais de idade, total e que tiveram filhos - Brasil 2010. [Internet]. 2010 [acesso em: 28 mai 2014]. Disponível: ftp://ftp.ibge.gov.br/Censos/Censo_ Demografico_2010/Nupcialidade_Fecundidade_ Migracao/tab1_2.pdf

6. Prefeitura Municipal de São Sebastião do Paraíso. Secretaria de Saúde e Ação Social. Vigilância em Saúde. DATASUS - SINASC - Sistema de Informação sobre Nascidos Vivos - Sistema Local. [Internet]. 2011 [acesso em: 13 dez 2011]. Disponível em: http://localhost/SINASC_LOCAL/rel_ocorr_resid. asp?pag_atual $=1 \&$ co_uf_ibge $=31 \&$ co_municipio_ ibge=316470\&tp_municipio $=\& s g$

7. Spindola T, Silva LFF. Perfil epidemiológico de adolescentes atendidas no pré-natal de um hospital universitário. Esc. Anna Nery. 2009; 13(1):99-107.

8. Santos JA, Silva CFS, Petenão E, Soster FCB, Berard $M B$, Silva SR. Perfil das adolescentes com reincidência de gravidez assistidas no setor público de Indaiatuba (SP). J. Health Sci. Inst. 2009; 27(2):115-21.
9. Meincke SMK, Oliveira MRP, Trigueiro DRSG, Carraro TE, Gondim ETC, Collet N. Perfil socioeconômico e demográfico de puérperas adolescentes. Cogitare enferm. 2011:16(3):486-91.

10. Ministério da Saúde (BR). Indicadores e Dados Básicos - Brasil - 2012. Proporção de nascidos vivos de mães adolescentes por Unidade da Federação. [Internet].2012 [acesso em: 28 mai 2014]. Disponível em: http://tabnet. datasus.gov.br/cgi/tabcgi.exe?idb2012/g15.def

11. Rodrigues MF, Jardim DP. Conhecimento e uso da contracepção de emergência na adolescência: contribuições para a enfermagem. Cogitare enferm. 2012:17(4):724-9.

12. Baratieri T, Vieira VCL, Marcon SS. A visão da adolescente com reincidência gestacional sobre a família. Esc. Anna Nery. 2011;15(2):261-9.

13. Taborda JA, Silva FC, Ulbricht L, Neves EB. Consequências da gravidez na adolescência para as meninas considerando-se as diferenças socioeconômicas entre elas. Cad. saude colet. 2014:22(1):16-24.

14. Instituto Brasileiro de Geografia e Estatística (IBGE). Indicadores Sociodemográficos e de Saúde no Brasil. Rio de Janeiro (RJ): Ministério do Planejamento; 2009. 152 p.

15. Santos MMAS, Balão MR, Barros DC, Pinto AA, Pedrosa PLM, Saunders C. Estado nutricional prégestacional, ganho de peso materno, condições da assistência pré-natal e desfechos perinatais adversos entre puérperas adolescentes. Rev. Bras. Epidemiol. 2012:15(1):143-54.

16. Madureira L, Marques IR, Jardim DP. Contracepção na adolescência: conhecimento e uso. Cogitare enferm. 2010:15(1):100-5.

17. Duarte HHS, Bastos GAN, Duca GFD, Cortela HVE. Utilização de métodos contraceptivos por adolescentes do sexo feminino da Comunidade Restinga e Extremo Sul. Rev. paul. pediatr. 2011;29(4):572-6.

18. Trajano MFC, Quirino GS, Gonçalves GAA. Consequências da maternidade na adolescência. Cogitare enferm. 2012:17(3):430-6.

19. Silva APF, Hirai KN, Silva ME, Hoeredia EP. Os fatores emocionais gerados pela gravidez na adolescência. Conscientiae Saúde. 2009;8(1):91-8.

20. Rodrigues DF, Rodrigues FRA, Silva LMS, Jorge MSB, Vasconcelos LDGP. O adolescer e ser mãe: representações sociais de puérperas adolescentes. Cogitare enferm. 2009:14(3):455-62.21- Bruno ZV, Feitosa FEL, Silveira KP, Morais IQ, Bezerra MF. Reincidência de gravidez em adolescentes. Rev. bras. ginecol. obstet. 2009;31(10):480-4. 